DOI: http://dx.doi.org/10.12957/demetra.2015.13376

\title{
Adequação do consumo e evolução antropométrica após educação nutricional de pacientes com diabetes mellitus tipo 2
}

\section{Fitness of intake and anthropometric after nutritional education of patients with type 2 diabetes mellitus}

\author{
Márcia Ferreira Cândido de Souza' \\ Viviane Ferreira Araúio² \\ 1 Universidade Federal de Sergipe, Núcleo de Pós \\ Graduação em Medicina. Aracaju-SE, Brasil. \\ ${ }^{2}$ Universidade Federal de Sergipe, Departamento de \\ Nutrição. Aracaju-SE, Brasil. \\ Correspondência / Correspondence \\ Márcia Ferreira Cândido de Souza \\ E-mail:marciacandido@ufs.br
}

\section{Resumo}

Introdução: Alguns autores defendem que a educação nutricional é o ponto chave para o controle dos índices glicêmicos e prevenção de complicações secundárias no diabetes mellitus tipo 2 (DM 2). O objetivo desse estudo foi analisar a adequação do consumo alimentar e a evolução antropométrica de pacientes diabéticos tipo 2, antes e após educação nutricional. Metodologia: Estudo transversal realizado com 32 pacientes participantes de grupos de educação nutricional portadores de DM 2, onde foram analisados dados antropométricos e o consumo alimentar, antes e após sessões de educação nutricional. O número de porções de alimentos consumidos foi classificado pela Pirâmide Alimentar Brasileira e pela pirâmide adaptada para diabéticos da American Diabetes Association. Resultados: A população do estudo foi composta em sua maioria $(90,6 \%)$ por mulheres. Não houve melhora de nenhum dos indicadores antropométricos entre os dois momentos da avaliação, assim como, não houve melhora no consumo dos grupos de alimentos que apresentaram inadequação antes da educação nutricional de acordo com as duas pirâmides, após a educação nutricional. A adequação do consumo segundo a Pirâmide da American Diabetes Association apresentou uma piora significativa para o grupo de grãos e fontes de carboidrato mais leguminosas $(\mathrm{p}=0,09)$ e para o grupo de gorduras, doces e álcool (p <0,01). Conclusão: Apesar do programa de educação nutricional avaliado não ter apresentado um resultado satisfatório no que se refere à melhora nos hábitos alimentares dos pacientes do estudo, o mesmo deve ser reavaliado e incentivado.

Palavras-chave: Hábitos Alimentares. Educação Alimentar e Nutricional. Diabetes Mellitus. 


\section{Abstract}

Introduction: Some authors argue that nutrition education is the key point for the control of blood glucose levels and prevention of secondary complications in diabetes mellitus type 2 (DM2). The aim of this study was to analyze the adequacy of food consumption and anthropometric development of type 2 diabetic patients before and after nutrition education. Methodology: Cross-sectional study with 32 patients in group nutrition education with DM2, where anthropometric data and dietary intake were analyzed before and after nutrition education sessions. The number of servings of food consumed was classified by the Brazilian Food Pyramid and pyramid adapted for diabetics by the American Diabetes Association. Results: The study population was composed mostly $(90.6 \%)$ women. There was no improvement in any of the anthropometric indicators between the two evaluation times, as there was no improvement in the consumption of food groups that had inadequate before nutrition education according to the two pyramids, after the nutrition education. The adequacy of intake according to Pyramid American Diabetes Association showed a significant worsening for the group of grains and carbohydrate sources more legumes $(\mathrm{p}=0.09)$ and for the group of fats, sweets and alcohol $(\mathrm{p}<0.01)$. Conclusion: Despite the nutritional education program evaluated not have presented a satisfactory result with regard to the improvement in dietary habits of the study patients, the same should be reassessed and encouraged.

Key words: Eating Habits. Food and Nutrition Education. Diabetes Mellitus.

\section{Introdução}

O diabetes mellitus tipo 2 (DM2) acomete, em média, 300 milhões de pessoas no mundo, e aproximadamente $20 \%$ dos indivíduos que possuem entre 65 e 76 anos são considerados diabéticos. ${ }^{1}$ No Brasil, a prevalência de diabetes chega a 7,6\% da população, sendo que quase metade das pessoas acometidas pela doença $(46 \%)$ desconhece o diagnóstico. ${ }^{2}$

Considera-se que mudanças nos hábitos alimentares de pacientes portadores de DM2, priorizando alimentos com baixo índice glicêmico, ricos em fibras e com baixas quantidades de gorduras, reduzem os níveis séricos de glicose e insulina no período pós-prandial. ${ }^{2}$ Alguns autores defendem que a educação nutricional é o ponto-chave para o controle dos índices glicêmicos e 
prevenção de complicações secundárias no DM2, visto que se torna impossível manter um controle metabólico sem uma alimentação adequada. ${ }^{3}$ Wing et al. ${ }^{4}$ forneceram, em seu estudo, evidências da eficácia de programas de estilo de vida de baixo custo na redução da incidência de diabetes mellitus tipo 2.

A necessidade de desenvolver atividades de ensino ou práticas educativas de saúde, direcionadas ao paciente diabético e a sua família, está relacionada à prevenção de complicações através do automanejo da doença, o que permite ao paciente conviver melhor com esse problma. ${ }^{5}$

As medidas antropométricas são importantes para avaliação do estado nutricional e evolução dos indivíduos diabéticos, já que auxiliam a monitorar as possíveis mudanças apresentadas e a traçar o tipo de tratamento dietético mais adequado. Dessa forma, ambas as medidas refletem a eficácia do tratamento e a adesão do paciente. ${ }^{6,7}$

Diante do exposto, o presente estudo teve como objetivo analisar a adequação do consumo alimentar de acordo com a Pirâmide Alimentar Brasileira ${ }^{8}$ e com a pirâmide adaptada para diabéticos da American Diabetes Association ${ }^{9}$ e a evolução antropométrica, antes e após educação nutricional de pacientes diabéticos tipo 2, assistidos pelo Ambulatório de Nutrição do Hospital Universitário de Sergipe (HU).

\section{Metodologia}

O estudo foi do tipo transversal com dados referentes à avaliação nutricional e ao consumo alimentar de pacientes participantes de grupos de educação nutricional portadores de DM2, assistidos no Ambulatório de Nutrição do Hospital Universitário de Sergipe. A população do estudo foi composta de adultos e idosos de ambos os sexos com diagnóstico de DM2.

Foram incluídos todos os dados referentes à avaliação nutricional e ao consumo alimentar de pacientes adultos e idosos portadores de diabetes mellitus tipo 2 coletados antes e após a participação dos mesmos nas sessões de educação nutricional realizadas pelos profissionais nutricionistas do Ambulatório de Nutrição do HU.

Foram excluídos da amostra pacientes que não se apresentaram na consulta de retorno após a educação nutricional e que, portanto, não possuíam os dados relativos à avaliação nutricional e ao consumo alimentar no protocolo de nutrição do ambulatório.

\section{Coleta de dados do consumo alimentar}

Foram coletados, dos protocolos do Ambulatório de Nutrição, dados do consumo alimentar através de recordatórios de 24 horas (R24h), antes da educação nutricional e na primeira consulta 
após a referida intervenção de pacientes do estudo. Para o cálculo das porções de alimentos, foi utilizado o software NutWin (2002) e para classificação do número de porções consumidas utilizaram-se como base a Pirâmide Alimentar Brasileira ${ }^{8}$ e a pirâmide adaptada para diabéticos da American Diabetes Association. ${ }^{9}$

\section{Coleta de dados antropométricos}

Foram coletados dos referidos protocolos dados relacionados a peso, estatura e circunferência abdominal de pacientes da amostra, registrados antes da educação nutricional e na primeira consulta após a referida intervenção.

\section{Avaliação da educação nutricional}

A educação nutricional avaliada neste estudo é realizada rotineiramente pelos profissionais nutricionistas do Ambulatório de Nutrição na assistência aos pacientes diabéticos como parte do tratamento nutricional. As sessões de educação nutricional consistem em aulas explicativas sobre alimentação do ponto de vista quantitativo de acordo com a Pirâmide Alimentar Brasileira $^{8}$ e oficinas de degustação sobre alimentação saudável.

Convém ressaltar que a pirâmide proposta pela American Diabetes Association ${ }^{9}$ foi utilizada pelos autores para análise da adequação do consumo da amostra pelo parâmetro específico de consumo para portadores de DM2, mas não foi utilizada como instrumento nas sessões de educação nutricional realizadas no Ambulatório de Nutrição.

\section{Análise estatística}

A análise dos dados foi realizada utilizando-se o programa Statistical Package for the Social Sciences (SPSS), versão 18.0. Foi utilizada a análise descritiva das variáveis do estudo pelo cálculo de médias, desvios-padrão e medianas, quando mais adequado. Para a comparação entre as variáveis, foram utilizados os testes t pareado, para dados paramétricos independentes, e o de Wilcoxon, para dados não paramétricos.

Para todas as análises estatísticas, foi adotado um nível de significância estatística de $5 \%$, ou seja, $\mathrm{p} \leq$ 0,05. O projeto foi aprovado pelo Comitê de Ética e Pesquisa do HU/UFS. 


\section{Resultados}

Foram avaliados 32 pacientes com DM2, participantes do projeto de educação nutricional em um ambulatório de nutrição. A população do estudo foi composta, em sua maioria (90,6\%), por mulheres. A faixa etária da amostra variou entre 22 a 85 anos, sendo a média de idade dos homens 56,7 $\pm 16,2$ anos e das mulheres, 53,3 \pm 17,8 anos. As comorbidades mais frequentes foram hipertensão arterial sistêmica $(65,6 \%)$, dislipidemia $(43,8 \%)$, doenças cardiovasculares $(15,6 \%)$ e câncer $(6,3 \%)$.

$\mathrm{Na}$ análise do perfil antropométrico, foi observado que a amostra apresentou média de IMC compatível com o diagnóstico nutricional de obesidade grau I e não houve melhora significativa entre nenhum dos indicadores antropométricos nos dois momentos da avaliação realizada (tabela 1).

Tabela 1. Análise do perfil antropométrico de pacientes com diabetes mellitus tipo 2 antes e após educação nutricional. Aracaju-SE, 2014.

\begin{tabular}{cccc}
\hline & Antes da & Depois da & \\
Indicadores Antropométricos & Educação & Educação & $\mathrm{p}^{* *}$ \\
& Nutricional & Nutricional & \\
& $\mathrm{X}+\mathrm{DP} *$ & $\mathrm{X}+\mathrm{DP}$ & \\
\hline Peso $(\mathrm{kg})$ & $78,9 \pm 21,9$ & $78,8 \pm 22,4$ & 0,76 \\
IMC $\left(\mathrm{kg} / \mathrm{m}^{2}\right)$ & $31,7 \pm 7,4$ & $31,6 \pm 7,6$ & 0,56 \\
Circunferência abdominal $(\mathrm{cm})$ & $104,8 \pm 16,7$ & $104,6 \pm 16,6$ & 0,70
\end{tabular}

*X + DP = Média + Desvio padrão.

**Teste t pareado $(\mathrm{p} \leq 0,05)$.

De acordo com a avaliação do consumo tendo como referência a Pirâmide Alimentar Brasileira ${ }^{8}$, após a educação nutricional não houve melhora no consumo dos grupos de alimentos que apresentaram inadequação antes da educação nutricional (tabela 2). 
Tabela 2. Análise do consumo dos grupos de alimentos antes e após educação nutricional de acordo com a Pirâmide Alimentar Brasileira. Aracaju-SE, 2014.

\begin{tabular}{ccccc}
\hline Grupos de Alimentos & $\begin{array}{c}\text { Antes da } \\
\text { Referência } \\
\text { (porções) }\end{array}$ & $\begin{array}{c}\text { Educação } \\
\text { Nutricional } \\
\text { (porções) } \\
\text { X + DP** }\end{array}$ & $\begin{array}{c}\text { Depois da } \\
\text { Educação } \\
\text { Nutricional } \\
\text { (porções) } \\
\text { X + DP** }\end{array}$ & p*** \\
\hline Pães e cereais & 5 a 9 & $3,7 \pm 2,0$ & $3,0 \pm 1,5$ & 0,15 \\
Frutas & 3 a 5 & $2,4 \pm 2,8$ & $2,8 \pm 3,5$ & 0,69 \\
Hortaliças & 4 a 5 & $1,8 \pm 2,5$ & $1,4 \pm 1,5$ & 0,97 \\
Leguminosas & 1 & $0,9 \pm 1,0$ & $1,1 \pm 1,2$ & 0,08 \\
Carnes e ovos & 1 a 2 & $1,7 \pm 1,7$ & $1,6 \pm 1,1$ & 0,81 \\
Leite e produtos lácteos & 3 & $0,6 \pm 0,8$ & $1,2 \pm 1,7$ & 0,19 \\
Açúcares e doces & 1 a 2 & $0,2 \pm 0,6$ & $0,3 \pm 0,8$ & 0,57 \\
Óleos e gorduras & 1 a 2 & $0,6 \pm 1,2$ & $0,3 \pm 0,7$ & 0,12 \\
\hline
\end{tabular}

* Pirâmide Alimentar Brasileira (Philippi et al., 1999).

$* * \mathrm{X}+\mathrm{DP}=$ Média + Desvio Padrão.

***Teste de Wilcoxon $(\mathrm{p} \leq 0,05)$.

Com relação à análise do consumo tendo como referência a Pirâmide da American Diabetes Association ${ }^{9}$, após a educação nutricional também não houve melhora no consumo dos grupos de alimentos que apresentaram inadequação antes da educação nutricional (tabela 3). 
Tabela 3. Análise do consumo dos grupos de alimentos antes e após educação nutricional de acordo com a Pirâmide da American Diabetes Association (2008). Aracaju-SE, 2014.

\begin{tabular}{ccccc}
\hline Grupos de Alimentos & $\begin{array}{c}\text { Referência } \\
\text { (porções) }\end{array}$ & $\begin{array}{c}\text { Antes da } \\
\text { Educação } \\
\text { Nutricional } \\
\text { (porções) } \\
\text { X + DP** }\end{array}$ & $\begin{array}{c}\text { Depois da } \\
\text { Educação } \\
\text { Nutricional } \\
\text { (porções) }\end{array}$ & p*** \\
X + DP** & \\
\hline $\begin{array}{c}\text { Grãos e fontes de } \\
\text { carboidrato mais }\end{array}$ & 6 a 11 & $4,4 \pm 2,4$ & $4,0 \pm 2,2$ & 0,31 \\
leguminosas & 2 a 4 & $2,4 \pm 2,8$ & $2,8 \pm 3,5$ & 0,69 \\
Frutas & & & & \\
Vegetais & 3 a 5 & $1,8 \pm 2,5$ & $1,4 \pm 1,5$ & 0,97 \\
Larnes e substitutos & 4 a 6 & $1,7 \pm 1,7$ & $1,6 \pm 1,1$ & 0,08 \\
Leite & 2 a 3 & $0,6 \pm 0,8$ & $1,2 \pm 1,7$ & 0,19 \\
Gorduras, doces e álcool & 1 a 2 & $0,9 \pm 1,2$ & $0,5 \pm 0,9$ & 0,26 \\
\hline
\end{tabular}

*Pirâmide da American Diabetes Association (2008).

** $\mathrm{X}+\mathrm{DP}=$ Média + Desvio padrão.

***Teste de Wilcoxon $(\mathrm{p} \leq 0,05)$.

Ao avaliar o percentual de participantes do estudo que, após a educação nutricional, adequaram o consumo dos grupos de alimentos para o número de porções determinadas pela Pirâmide Alimentar Brasileira (tabela 4), observou-se aumento significativo no número de participantes que melhoraram o consumo de leguminosas $(\mathrm{p}<0,01)$ e redução significativa no número de participantes que consumiam adequadamente o grupo de pães e cereais ( $p=0,03$ ). 
Tabela 4. Adequação do consumo dos grupos de alimentos antes e após educação nutricional, segundo a Pirâmide Alimentar Brasileira. Aracaju-SE, 2014.

\begin{tabular}{cccc}
\hline Grupos de Alimentos & $\begin{array}{c}\text { Antes da Educação } \\
\text { Nutricional }\end{array}$ & $\begin{array}{c}\text { Depois da Educação } \\
\text { Nutricional }\end{array}$ & \\
\hline Consumo & Consumo Adequado & $\mathrm{p}^{*}$ \\
Pdequado (\%) & $(\%)$ & 0,03 \\
Frutas & 28,1 & 9,4 & 0,53 \\
Hortaliças & 31,3 & 25,0 & 0,16 \\
Leguminosas & 15,6 & 9,4 & $<0,01$ \\
Carnes e ovos & 6,3 & 28,1 & 0,40 \\
Leite e produtos & 47,0 & 37,5 & 1,00 \\
lácteos & 0,0 & 0,0 & 0,56 \\
Açúcares e doces & 3,1 & 6,3 & 0,16 \\
Óleos e gorduras & 9,4 & 3,1 & \\
\hline
\end{tabular}

*Teste de Wilcoxon $(\mathrm{p} \leq 0,05)$.

De acordo com a Pirâmide da American Diabetes Association ${ }^{9}$, o percentual de participantes do estudo que, após a educação nutricional, adequaram o consumo dos grupos de alimentos para o número de porções determinadas pela mesma (tabela 5) piorou significativamente para o grupo de grãos e fontes de carboidrato mais leguminosas $(\mathrm{p}=0,09)$ e para o grupo de gorduras, doces e álcool ( $\mathrm{p}<0,01)$. 
Tabela 5. Adequação do consumo dos grupos de alimentos antes e após educação nutricional segundo a Pirâmide da American Diabetes Association (2008). Aracaju-SE, 2014.

\begin{tabular}{cccc}
\hline Grupos de Alimentos & $\begin{array}{c}\text { Antes da } \\
\text { Educação } \\
\text { Nutricional }\end{array}$ & $\begin{array}{c}\text { Depois da } \\
\text { Educação } \\
\text { Nutricional }\end{array}$ & \\
& Consumo & Consumo & $\mathrm{p}^{*}$ \\
Adequado (\%) & Adequado (\%) & \\
Grãos e fontes de carboidrato & 34,4 & 18,8 & 0,09 \\
mais leguminosas & & & 0,37 \\
Frutas & 28,1 & 37,5 & 0,56 \\
Vegetais & 18,8 & 15,6 & 0,56 \\
Carnes e substitutos & 6,3 & 3,1 & 0,32 \\
Leite & 9,4 & 3,1 & $<, 01$ \\
\hline
\end{tabular}

*Teste de Wilcoxon $(\mathrm{p} \leq 0,05)$.

Apesar de os pacientes terem melhorado a adequação do consumo de leguminosas de acordo com a Pirâmide Alimentar Brasileira, ${ }^{8}$ o fato de as leguminosas estarem incluídas no grupo de grãos e fontes de carboidratos na Pirâmide da American Diabetes Association ${ }^{9}$ fez com que seu consumo tenha piorado na análise da adequação, segundo a referida pirâmide. $\mathrm{O}$ consumo de pães e cereais na Pirâmide Alimentar Brasileira, ${ }^{8}$ que equivale ao grupo de grãos e fontes de carboidratos mais leguminosas na Pirâmide da American Diabetes Association, ${ }^{9}$ também apresentou piora significativa em seu consumo, conforme demonstrado anteriormente.

A representação gráfica da Pirâmide Alimentar Brasileira, ${ }^{8}$ com as distorções do consumo apresentadas ao final da educação nutricional, está representada na (figura 1). 


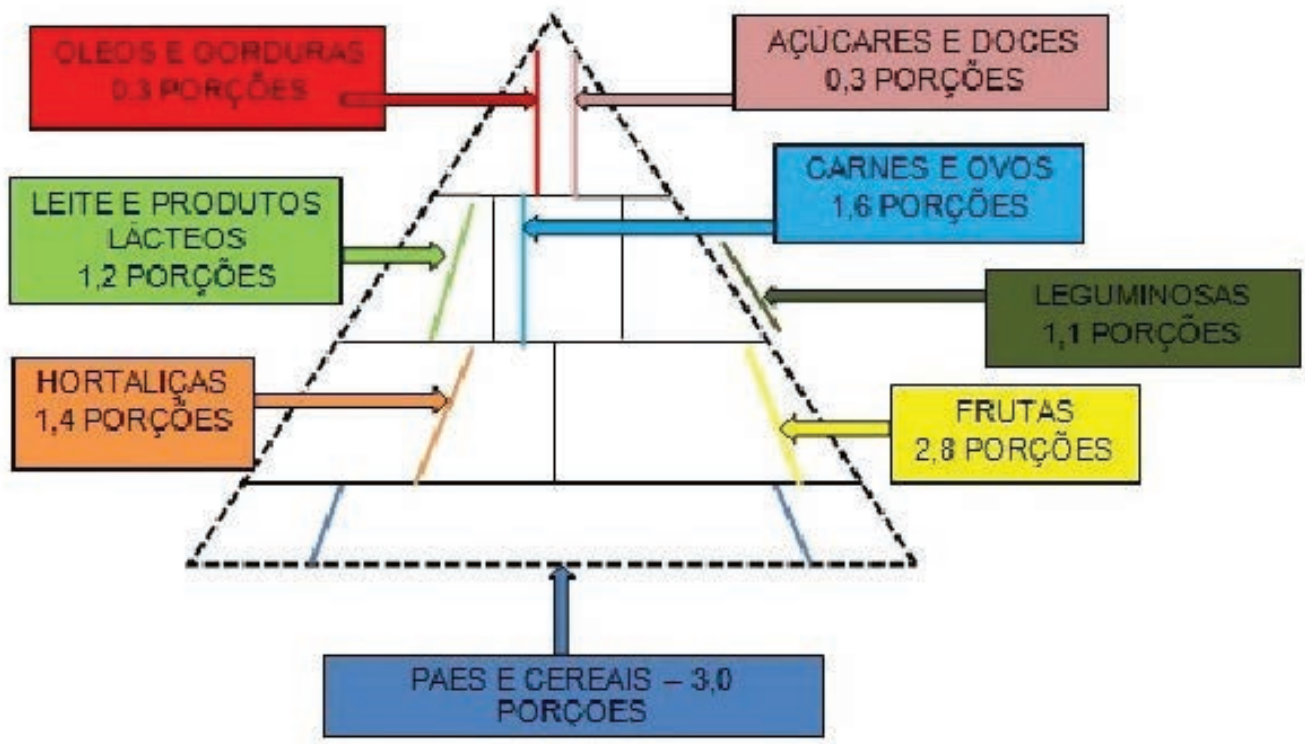

Figura 1. Pirâmide Alimentar Brasileira com as distorções do concurso após a educação nutricional.

\section{Discussão}

A prevalência do DM vem crescendo mundialmente, configurando-se atualmente como uma epidemia resultante, em sua maioria, do envelhecimento da população. Entretanto, a alimentação inadequada, o sedentarismo e o aumento da obesidade também são responsáveis pela expansão global da doença. ${ }^{10}$

A maior prevalência de mulheres observada no presente estudo também foi encontrada por Lima-Costa et al., ${ }^{11}$ ao avaliarem a validade de diabetes autorreferido e seus determinantes em 1.492 indivíduos, na cidade de Bambuí, Minas Gerais, em 2007, apresentando em seu estudo número quatro vezes maior de mulheres que de homens. Goldenberg et al., ${ }^{12}$ ao avaliarem a prevalência de DM, a diferença de gêneros e a igualdade entre os sexos em 2.007 indivíduos, encontraram maior presença da doença em mulheres (56,9\%).

Essa situação pode ser atribuída ao fato de as mulheres parecerem procurar auxílio médico com maior frequência e de forma mais precoce que os homens. ${ }^{13}$ Essa ideia também é reafirmada por Pereira et al., ${ }^{14} \mathrm{em}$ seu estudo com adultos diabéticos de ambos os sexos. 
As doenças associadas mais prevalentes foram hipertensão arterial sistêmica, dislipidemia e doenças cardiovasculares. Segundo Scheffel et al., ${ }^{15}$ os pacientes com DM2 têm uma propensão de mortalidade duas a quatro vezes maior por doença cardíaca em relação a não diabéticos, e quatro vezes mais chances de desenvolver doença vascular periférica (DVP) e acidente vascular cerebral (AVC).

A análise do perfil antropométrico dos pacientes do estudo mostrou que a maioria dos pacientes foi classificada como obesos e que não houve melhora significativa entre os dois momentos da avaliação em nenhum dos indicadores antropométricos pesquisados. Em estudo transversal ${ }^{16}$ realizado em Ribeirão Preto (SP), foi encontrada alta prevalência (91\%) de sobrepeso e obesidade entre os pacientes com diabetes tipo 2. Estima-se que $80 \%$ dos pacientes diabéticos apresentem obesidade ou excesso de peso. ${ }^{17}$

Silveira ${ }^{18}$ observou, em seus resultados, aumento da glicemia paralelo à elevação do valor do IMC, relacionado ao aumento da resistência à insulina. Os benefícios da perda de peso após intervenções sobre os estilos de vida foram demonstrados em vários estudos, com diminuição da hemoglobina glicada, triglicerídeos, marcadores de inflamação e insulinorresistência, pressão diastólica e da circunferência da cintura e com aumento dos níveis de HDL. ${ }^{19-21}$

A população do estudo não apresentou consumo adequado de porções dos grupos de alimentos de acordo com a Pirâmide Alimentar Brasileira ${ }^{8}$ e com a Pirâmide da American Diabetes Association ${ }^{9}$ em nenhum dos momentos da avaliação realizada. No entanto, após a educação nutricional, aumentou significativamente o percentual de pacientes que melhoraram o consumo do grupo de leguminosas de acordo com a Pirâmide Alimentar Brasileira. ${ }^{8}$

Alguns estudos indicam o quanto é difícil obter resultados satisfatórios em diabéticos, seja pela baixa redução de peso ou até aumento ponderal, da circunferência abdominal e da hemoglobina glicada. Tais situações evidenciam o grande desafio em relação à mudança de hábitos alimentares visando à perda ponderal e um bom controle metabólico. ${ }^{22}$

Em metanálise ${ }^{23}$ na qual foram analisados 37 artigos sobre o tema "saúde e doença versus diabetes" nas mais variadas formas, foram retratadas as dificuldades diárias sentidas pelo portador de DM e por seus familiares para o controle da doença. Tais dificuldades podem influenciar diretamente na adesão do portador de DM ao tratamento prescrito. Assim, os fatores comportamentais e emocionais apresentados pelo paciente devem ser considerados no planejamento de ações de saúde para a assistência integral a essa população.

Zanettiet et al. ${ }^{24}$ avaliaram, em seu estudo, a implementação do protocolo Staged Diabetes Management (SDM) no Centro Educativo de Enfermagem para Adultos e Idosos (CEEAI) da Escola de Enfermagem de Ribeirão Preto (EERP-USP). O estudo foi realizado durante um ano e 
os autores identificaram a existência de um grande desafio para a equipe multiprofissional que acompanhou os pacientes, tanto em relação à capacitação em educação em diabetes, quanto em relação à compreensão de que a aquisição do conhecimento não se traduz necessariamente em mudança de comportamento.

No entanto, apesar das dificuldades, os programas de educação para pacientes diabéticos devem ser incentivados, pois ajudam na otimização do controle metabólico, prevenção e controle das complicações e melhoram a qualidade de vida em relação a custo-efetividade. ${ }^{22}$

Considerando-se que a orientação nutricional é importante para auxiliar o indivíduo a melhorar seus hábitos alimentares e obter um controle metabólico da doença, ${ }^{25}$ essa deve ser realizada logo ao se fazer o diagnóstico de DM. É importante que a orientação dietética seja feita por profissional tecnicamente habilitado para desenvolver programas e ações de educação nutricional. ${ }^{11,26}$

Por fim, um fator limitante do estudo, e que também é importante discutir, é o sub-relato do consumo alimentar durante a anamnese alimentar, por parte dos pacientes estudados. Scagliusi \& Lancha ${ }^{27}$ explicam que o sub-relato é um elemento bastante complexo, envolvendo fatores morais, emocionais, sociais, físicos e cognitivos, que comprometem de forma significativa as deduções feitas a partir de estudos de avaliação de consumo alimentar. Segundo os autores, ${ }^{27}$ mulheres subrelatam seu consumo alimentar mais que os homens. Isso se dá pelo fato de esse grupo sofrer mais pressão social em relação à imagem corporal, o que as levaria a relatar um consumo alimentar considerado saudável, e não o real.

\section{Conclusão}

Apesar de o programa de educação nutricional avaliado não ter apresentado resultado satisfatório no que se refere à melhora nos hábitos alimentares dos pacientes do estudo, o mesmo deve ser reavaliado e incentivado, pois a educação nutricional é decisiva na manutenção de uma alimentação adequada e, consequentemente, no controle metabólico e prevenção de complicações no DM2.

\section{Agradecimentos}

Ao Ambulatório de Nutrição do Hospital Universitário de Sergipe (HU), por conceder os dados dos protocolos dos pacientes para a realização deste estudo. 


\section{Referências}

1. Oliveira PP, Fachin SM, Tozatti J, Ferreira MC, Marinheiro LPF. Análise comparativa do risco de quedas entre pacientes com e sem diabetes mellitus tipo 2. Rev. Assoc. Med. Bras. 2012; 58(2):234-239.

2. Carvalho FS, Netto AP, Zach P, Sachs A, Zanella MT. Importância da orientação nutricional e do teor de fibras da dieta no controle glicêmico de pacientes diabéticos tipo 2 sob intervenção educacional intensiva. Arq. Bras. Endocrinol. Metab. 2012; 56(2):110-119.

3. Oliveira PB, Franco LJ. Consumo de adoçantes e produtos dietéticos por indivíduos com diabetes melito tipo 2, atendidos pelo Sistema Único de Saúde em Ribeirão Preto, SP. Arq. Bras. Endocrinol. Metab. 2010; 54(5):455-62.

4. Wing RR, Venditti E, Jakicic JM, Polley BA, Lang W. Lifestyle intervention in overweight individual with a family history of diabetes. Diabetes Care 1998; 21(3):350-9.

5. Otero LM, Zanetti ML, Ogrizio MD. Knowledge of diabetic patients about their disease before and after implementing a diabetes education program. Rev. Latino-Am. Enferm. 2008; 16(2):231.

6. Geraldo JM, colaboradores. Intervenção nutricional sobre medidas antropométricas e glicemia de jejum de pacientes diabéticos. Rev. Nutr. 2008; 21(3):329-340.

7. Castro SH, Matos HJ, Gomes MB. Parâmetros Antropométricos e Síndrome Metabólica em Diabetes Tipo 2. Arq. Bras. Endocrinol. Metab. 2006; 50(3):450-455.

8. Philippi ST, Latterza AR, Cruz ATR, Ribeiro LC. Pirâmide alimentar adaptada: guia para escolha dos alimentos. Rev. Nutr. 1999; 12(1):65-80.

9. American Diabetes Association. Nutrition recommendations and interventions for diabetes: a position statement of the American Diabetes Association. Diabetes Care 2008; 31(Supl. 1):S61-S78.

10. Rosa RS, Schmidt MI. Diabetes mellitus: magnitude das hospitalizações na rede pública do Brasil, 1999-2001. Epidemiol. Serv. Saúde 2008; 17(2):123-153.

11. Lima-Costa MF, colaboradores. Validade do diabetes auto-referido e seus determinantes: evidências do projeto Bambuí. Rev. Saúde Pub. 2007; 41(6):947-953.

12. Goldenberg P, Schenkman S, Franco LJ. Prevalência de diabetes mellitus: diferenças de gênero e igualdade entre os sexos. Rev. Bras. Epidemiol. 2003; 6(1):18-28.

13. Jonsson PM, Sterky G, Gafvels C, Ostman J. Gender equity in health care: the case of Swedish diabetes care. Health Care Women Int. 2000; 21(5):413-31.

14. Pereira LKC, Farias RMP, Lourenço VV. Comportamento alimentar dos pacientes diabéticos atendidos pelo programa de atenção ao diabético de Hospital Universitário de Belém, PA. Rev. Nutr. Paut. 2007; 23(5):24-28.

15. Scheffel RS, Bortolanza D, Weber CS, Costa LA, Canani LH, Santos KG, et al. Prevalência de complicações micro e macrovasculares e de seus fatores de risco em pacientes com Diabetes Melito do Tipo 2 em atendimento ambulatorial. Rev. Assoc. Med. Bras. 2004; 50(3):263-267.

16. Oliveira KCS. Conhecimento e atitude de usuários com diabetes mellitus tipo 2 e hipertensão arterial em uma unidade básica de saúde de Ribeirão Preto, SP [Tese]. São Paulo: Universidade de São Paulo; 2009. 
17. Feldstein AC, Nichols GA, Smith DH, Stevens VJ, Bachman K, Rosales AG, et al. Weight change in diabetes and glycemic and blood pressure control. Diabetes Care 2008; 31(10):1960-5.

18. Silveira LAG. Correlação entre Obesidade e Diabetes Tipo 2. Juiz de Fora-MG: Pós-graduação Latu-Sensu em Fisiologia do Exercício e Avaliação-Morfofuncional Universidade Gama Filho; 2002.

19. Vetter ML, Wadden TA, Chittams J, Diewald LK, Panigrahi E, Volger S, et al. Effect of lifestyle intervention on cardiometabolic risk factors: results of the POWER- UP Trial. Int. J. Obes. 2013; 37(Supl. 1):S19-S24.

20. Espeland MA, Rejeski WJ, West DS, Bray GA, Clark JM, Peters AL, et al. Intensive weight loss intervention in older individuals: results from the Action for Health in Diabetes Type 2 diabetes mellitus trial. J. Am. Geriatr. Soc. 2013; 61(6):912-22.

21. Unick JL, Beavers D, Bond DS, Clark JM, Jakicic JM, Kitabchi AE, et al. The longterm effectiveness of a lifestyle intervention in severely obese individuals. Am. J. Med. 2013; 126(3):236-42.

22. Watanabe JA, Ávila ALVE, Isosaki M, Vieira LP, Girardi P, Takiuti ME. Avaliação de pacientes diabéticos portadores de doença arterial coronariana participantes de um programa de controle de peso. Rev. Bra. Med. 2013; 70(4):129-134.

23. Costa JA, Balga RSM, Alfenas RCG, Cotta RMM. Promoção da saúde e diabetes: discutindo a adesão e a motivação de indivíduos diabéticos participantes de programas de saúde. Ciênc. Saúde Col. 2011; 16(3):2001-2009.

24. Zanetti ML, Otero LM, Freitas MCF, Santos MA, Guimarães FPM, Couri CEB, et 1. Atendimento ao paciente diabético utilizando o protocolo Staged Diabetes Management: relato de experiência. Rev. Bra. Prom. Saúde 2006; 19(4):253-260.

25. American Diabetes Association. Standards of medical care in diabetes. Diabetes Care 2010; 33(1):S11-S61.

26. Fontinele RSS, Peres LCL, Nascimento MAB, Boni MS. Avaliação do conhecimento sobre alimentação entre pacientes com diabetes tipo 2. Com. Cienc. Saúde 2007; 18(3):197-206.

27. Scagliusi FB, Lancha-Junior AH. Subnotificação da ingestão energética na avaliação do consumo alimentar. Rev. Nutr. 2003; 16(4):471-481.

Recebido: $22 / 10 / 2014$

Revisado: $15 / 12 / 2014$

Aprovado: 05/1/2015 